The following contributions have been accepted for publication and will appear in 1980

Stuart S. Antman

Nonlinear eigenvalue problems for the whirling of heavy elastic strings P. R. BEESACK

A simpler proof of two inequalities of Brodlie and Everitt

Patrick J. Browne and B. D. Sleeman

Stability regions for multi-parameter systems of periodic second order ordinary differential equations

J. Duncan and S. A. R. Hosseiniun

The second dual of a Banach algebra

NELSON DUNFORD

Some ergodic theorems

J. B. Fountain and P. LockLey

Bands with distributive congruence lattice

A. Haraux

Comportement à l'infini pour certains systèmes dissipatifs non linéairs Einar Hille

On a class of interpolation series

K. KRerth and C. A. Swanson

Higher order Wirtinger inequalities

Pierre-Louis Lions

Problèmes elliptiques du 2ème ordre non sous forme divergence

David Race

On the location of the essential spectra and regularity fields of complex Sturm-Liouville operators

Christine ThurLow

A generalisation of the inverse spectral theorem of Levitan and Gasymov The point-continuous spectrum of second-order, ordinary differential operators 
CONTENTS

\section{ERICH MARTENSEN}

The Rothe method for the wave equation in several space dimensions

G. F. WeBB

Compactness of bounded trajectories of dynamical systems in infinite dimensional spaces

Peter Wolfe

On diffracted wave fronts

Allan M. Sinclair

Cohen elements in Banach algebras

F. KAPPEL and W. SHAPPACHER

Non-linear functional differential equations and abstract integral equations

A. J. KNIGHT

Some loci in $S_{5}$ associated with systems of conics in a plane

A. J. KNIGHT

Conics related by a pentogram: a problem of J. E. Reeve

Don B. Hinton and Roger T. Lewis

Singular differential operators with spectra discrete and bounded below

David Colton

The inverse scattering problem for a cylinder

Herbert amann and Peter Hess

A multiplicity result for a class of elliptic boundary value problems

N. CurLe

Effects of a sharp pressure rise on a compressible laminar boundary layer, when the Prandtl number is $\sigma=0.72$

Alastair D. Wood

On the deficiency indices of a fourth order singular differential operator

Published by The Royal Society of Edinburgh

22 George Street, Edinburgh EH2 2PQ

Printed in Northern Ireland at The Universities Press (Belfast) Ltd. 